\title{
LA FORMALIZACIÓN, FINANCIAMIENTO, CAPACITACIÓN, COMPETITIVIDAD Y LA RENTABILIDAD EN LAS MICRO Y PEQUEÑAS EMPRESAS, CHIMBOTE, 2010-2013
}

\section{THE FORMALIZATION, FINANCING, TRAINING, COMPETITIVENESS AND PROFITABILITY IN MICRO AND SMALL ENTERPRISES, CHIMBOTE, 2010- 2013}

\author{
Reinerio Zacarías Centurión Medina*
}

\begin{abstract}
RESUMEN
E $^{l}$ presente estudio tuvo como objetivo determinar las principales características de la formalización, el financiamiento, la capacitación, la competitividad y la rentabilidad, de las micro y pequeñas empresas del distrito de Chimbote, periodo 2010-2013, de diseño no experimental-transversal-descriptivo. Se utilizó un muestreo no probabilístico intencional. De una población de 756 micro y pequeñas empresas (mype), se extrajo una muestra dirigida de 272 mype, en las cuales se determinaron las principales características de las micro y pequeñas empresas y de sus representantes; en 120 mypes se determinó las características de la formalización; en 196 mypes, del financiamiento; en 77 mypes, de la capacitación; en 78 mypes, de la competitividad, y en 128 mypes, de la rentabilidad, todas las cuales se presentan en tablas simples de frecuencias absolutas. Se utilizó como instrumento un cuestionario estructurado, aplicado a los representantes de las mypes mediante la técnica de la encuesta. Se llegó a las siguientes conclusiones: La mayoría de las mypes del distrito de Chimbote, periodo 2010-2013, tienen una permanencia de 4 años a más de 7 años; son formales y fueron creadas para obtener ganancias. La formalización les proporciona ventajas como obtención de créditos, aumento de sus ventas y les ayuda a posicionarse en el mercado; en el financiamiento las mypes han solicitado créditos de 1 a 5 veces, pagando una tasa de más del $20 \%$ anual, invirtiéndolo en capital de trabajo; en la capacitación, las mypes consideran que esta es una inversión, y ha capacitado a su personal más de una vez en marketing y atención al cliente; en la competitividad, la mayoría de las mypes cuenta con un plan estratégico, conoce a sus competidores y percibe que es competitivo. Por último, con respecto a la rentabilidad
\end{abstract}

\footnotetext{
* Doctor en Administración. Docente, tutor, investigador, asesor de tesis en la Escuela Profesional de Administración de la Universidad Católica Los Ângeles de Chimbote. Correo electrónico: reyzack@hotmail.com
} 
se percibe que la mype es rentable y, por lo mismo, con esa rentabilidad ha mejorado su infraestructura.

Palabras clave: formalización, financiamiento, capacitación, competitividad, rentabilidad, micro y pequeñas empresas.

\section{ABSTRACT}

The present study took as a target to determine the main characteristics of the formalization, the financing, the training, the competitiveness and the profitability, of the micro and small enterprises of the district of Chimbote, period 2010-2013, of not experimental design - transversely - descriptive, a sampling was used not probabilístico intentionally. Of a population of 756 micro and small enterprises (MSE), a guided sample of 272 MSE was extracted; in which there decided the main characteristics of the micro and small enterprises and of its representatives; in 120 MSE it was determined the characteristics of the formalization, in 196 MSE of the financing, in 77 MSE of the training, in 78 MSE of the competitiveness, and in 128 MSE the characteristics of the profitability which appear in simple stage of absolute frequencies. It was used a structured questionnaire applied to the representatives of the MSE by means of the skill of the survey, coming to the following conclusions: Most of the MSE of the District of Chimbote period 2010-2013, have a permanence of four years to more than seven years; they are formal and were created for profit, in formalizing provides advantages such as obtaining credit, increased sales and helping them possession in the market, in financing MSEs have applied for loans from one to five times, paying a rate of more than $20 \%$ annually by investing in working capital, training MSE consider that it is an investment, and has trained its staff more than once in marketing and customer support, in competitiveness, most MSEs have a strategic plan, know your competitors and perceived to be competitive with respect to profitability perceives that the MSE is profitable and therefore that this profitability has improved its infrastructure.

Keywords: Formalizing, financing, training, competitiveness, profitability, Micro and Small Enterprises (MSE)

\section{INTRODUCCIÓN}

El país y el mundo avanzan a una velocidad acelerada hacia la globalización de la economía, la cultura y todas las esferas del quehacer de la humanidad. Esta situación plantea grandes retos a los países y a las micro y pequeñas empresas (mype), en cuanto a diversos temas, como la generación del empleo, mejora de la competitividad y, sobre todo, el crecimiento del país, lo cual generará que se pueda reducir, sustantivamente, la mitad de la pobreza, y una octava parte de extrema pobreza o las altas tasas de analfabetismo que actualmente tenemos. Ello quiere decir que es posible promover el desarrollo humano en nuestro país, teniendo a las mypes como un componente clave (Vela, 2007). Las mypes son las unidades económicas constituidas por una persona natural o jurídica, bajo cualquier forma de organización o gestión empresarial contemplada en la legislación vigente, 
que tiene como objeto desarrollar actividades de extracción, transformación, producción, comercialización de bienes o prestación de servicios (Vigil, 2008).

Pero a pesar de que las mypes ayudan al crecimiento y desarrollo de la economía de los países, estos a traviesan problemas que impiden su crecimiento y expansión en el mercado, tales como la formalización, financiamiento, capacitación, competitividad y rentabilidad en las mypes. En América Latina y, sin duda para casi todas las mypes, se cubren con capital propio y de reinversión, dadas las limitaciones de acceso a los servicios financieros. En este sentido, es destacable el rol de las agencias internacionales de cooperación que han puesto a prueba diversas modalidades de apoyo y modelos de organización para las mypes en áreas, tales como la formulación y ejecución de políticas locales, los servicios de desarrollo empresarial, el apoyo financiero para el microcrédito, la transferencia de tecnología, el desarrollo de nuevos productos, el mejoramiento de la competitividad y el desarrollo de mercados. Las mypes en el Perú constituyen un elemento clave para el desarrollo económico y social del país, sin embargo, aún no han superado el $5 \%$ de las colocaciones del sistema financiero nacional ni tampoco han superado las barreras de la formalidad a pesar que tienen bajo su responsabilidad más del $80 \%$ del empleo productivo. Esta débil situación se irá superando en la medida que la legislación laboral y tributaria sea un real motivador de la formalidad para que el sistema financiero pueda orientar los US\$ 9500 millones de liquidez disponibles (Arruñada, 2007).

Según Vélez (2010), las mypes no se formalizan por la complejidad del proceso en sí, ya que son procedimientos largos y muy complicados; son muchas las instituciones a las cuales se debe acudir para obtener licencias o permisos, todo lo cual genera altos costos por el tiempo que tienen que invertir en informarse y acudir a cada institución para hacer los trámites necesarios. Esto los desalienta, ya que no perciben los beneficios ante tales esfuerzos, es decir, el cumplimiento de los requisitos para la formalización distrae al pequeño inversionista, restándole competitividad y, lo que es peor, ganas de formalizarse.

Otro de los factores restrictivos para el desarrollo de las mypes se encuentra en el limitado acceso al financiamiento, la ausencia de una adecuada gestión financiera y administrativa para la toma de decisiones que generen valor económico; es decir, a pesar de que son las grandes generadoras de empleo y aportan el $64 \%$ del Producto Bruto Interno; al menos, el $15 \%$ de ellas obtienen financiamiento mediante la banca tradicional a tasas de interés superiores al $40 \%$, lo que implica mayores costos financieros y menores posibilidades de desarrollo, pero más del $80 \%$ de las pymes latinoamericanas no tienen acceso a ningún tipo de financiamiento debido a que no son consideradas sujetos de crédito (Torres, 2010). A esto se le suman los problemas de capacitación, tanto de la gerencia como del personal, lo que trae como consecuencia negativa que no alcancen la eficacia y eficiencia laboral en los puestos que desempeñan; es por esto que las mypes desaparecen con facilidad, pues la mayoría del personal de las mypes no están capacitadas, lo cual limita su participación en el mercado, según lo manifiesta Ponjuán (2010).

Asimismo, el Consejo Regional de las Mype de Áncash, con la colaboración y participación de sus miembros integrantes, han elaborado un Plan de Promoción y formalización para la competitividad y Desarrollo de las Mype, el cual constituye un documento de 
gestión con la finalidad de promover el desarrollo, la formalización y la competitividad de las mypes en nuestra región (Tantas 2010).

En Chimbote, donde se ha desarrollado la investigación existen un gran número de mypes en los diferentes sectores de servicio, comercio o producción, los cuales algunos desaparecen al corto tiempo de su creación y otros permanecen en el tiempo y se desarrollan en el mercado, desconociéndose el motivo de este fenómeno, considerándose que existen muchos factores como la formalización, el financiamiento, la capacitación, la competitividad y la rentabilidad en las mypes, los cuales por desconocimiento de parte de los representantes de estas, no son utilizados adecuadamente.

Todo lo señalado nos lleva a plantearnos la siguiente pregunta de investigación:

¿Cuáles son las principales características de la formalización, el financiamiento, la capacitación, la competitividad y la rentabilidad en las mypes del distrito de Chimbote, periodo 2010-2013? Para responder a esta pregunta de investigación se planteó como objetivo general determinar las principales características de la formalización, el financiamiento, la capacitación, la competitividad y la rentabilidad en las mypes del distrito de Chimbote, periodo 2010-2013. Y como objetivos específicos,

- Determinar las principales características de los representantes de las mypes del distrito de Chimbote, periodo 2010-2013.

- Determinar las principales características de las mypes del distrito de Chimbote, periodo 2010-2013.

- Determinar las principales características de la formalización en las mypes del distrito de Chimbote, periodo 2010-2013.

- Determinar las principales características del financiamiento en las mypes del distrito de Chimbote, periodo 2010-2013.

- Determinar las principales características de la capacitación en las mypes del distrito de Chimbote, periodo 2010-2013.

- Determinar las principales características de la competitividad en las mypes del distrito de Chimbote, periodo 2010-2013.

- Determinar las principales características de la rentabilidad en las mypes del distrito de Chimbote, periodo 2010-2013.

Esta investigación se justifica porque permitió obtener las principales características de la formalización, financiamiento, capacitación, competitividad y la rentabilidad que se aplican en las mypes para su crecimiento y desarrollo, dinamizando así el crecimiento económico y la mejora en la calidad de vida de la población, minimizando en cierta forma los índices de extrema pobreza en la cual se encuentra algunos sectores del distrito de Chimbote. Además, se justifica porque permitirá beneficiar con este conocimiento, el uso adecuado de las variables en estudio en las mypes, las cuales permanecerán en el mercado generando puestos de trabajo que beneficiará a la sociedad y, por último, servirá como base para realizar otras investigaciones. 


\section{MÉTODOS Y TÉCNICAS}

\section{Diseño de la investigación}

Fue un estudio que se desarrolló mediante un diseño no experimental de corte transversal-descriptivo.

\section{Población y muestra}

De una población de 756 mype las cuales se obtuvieron por los tesistas mediante sondeo, se extrajo una muestra dirigida de 272 mype del distrito de Chimbote, periodo 20102013 es decir se consideró como muestra a las micro y pequeñas cuyos representantes proporcionaron la información requerida. La unidad de análisis fue las micro y pequeñas empresas del distrito de Chimbote.

\section{Técnicas e instrumentos}

En el presente estudio se utilizó la técnica de la encuesta y como instrumento, el cuestionario estructurado. Fueron utilizados en la elaboración de las tesis materia de este metaanálisis.

\section{Plan de análisis}

De acuerdo con la naturaleza de la investigación, para el análisis de los datos se utilizó la técnica de análisis descriptivo y comparativo, como instrumentos el uso de tablas de distribución de frecuencias porcentuales. El procesamiento de los datos fueron tabulados en una matriz utilizando Microsoft Office Word/Excel 2007.

\section{Procedimiento}

Para el presente metaanálisis se recogió la información de todas las tesis que han desarrollado los tesistas en los talleres cocurriculares de investigación. La consolidación de la información permitió procesar los datos con el programa Excel para elaborar las tablas de frecuencia absoluta y relativa, ya que este programa cuenta con funciones de conteo sistemático, estableciéndose para ello criterios de conteo predeterminados. Después, se elaboraron las figuras circulares que se presentarán como anexo y que ayudaron a describir las variables. Estas figuras permitieron visualizar la distribución de los datos en las categorías que fueron objeto del análisis dentro de la investigación.

\section{Principios éticos}

El presente informe de metanaálisis se realizó bajo los principios de confiabilidad, confidencialidad, y respeto a la persona humana, utilizadas en la elaboración de las tesis materia de este estudio.

Confiabilidad, porque los datos utilizados fueron reales y obtenidos de libros, revistas, periódicos, internet, etc. A su vez, se obtuvo información de los trabajos de investigación 
realizados por los tesistas, quienes recibieron información de los representantes de las micro y pequeñas empresas en estudio.

Confidencialidad, porque solo se publicó la información que los representantes de las micro y pequeñas empresas involucradas en la investigación autorizó, guardando absoluta discreción de sus secretos empresariales sin propagar ninguna información confidencial, que dañe el normal desempeño de la actividad empresarial.

Respeto a la persona humana, porque se respetó las ideas, comentarios, información y opiniones que se virtieron durante la obtención de la información del trabajo de campo en la investigación.

\section{RESULTADOS}

Tabla 1. Características del representante de las mypes del distrito de Chimbote, periodo 2010- 2013.

\begin{tabular}{|c|c|c|}
\hline Datos generales: & Número de mype & Porcentaje \\
\hline \multicolumn{3}{|l|}{ Edad (años) } \\
\hline $18-30$ & 74 & 27,0 \\
\hline $31-50$ & 140 & 51,6 \\
\hline 51 a más & 58 & 21,4 \\
\hline Total & 272 & 100,0 \\
\hline \multicolumn{3}{|l|}{ Sexo } \\
\hline Masculino & 173 & 63,6 \\
\hline Femenino & 99 & 36,4 \\
\hline Total & 272 & 100,0 \\
\hline \multicolumn{3}{|l|}{ Grado de instrucción } \\
\hline Sin instrucción & 2 & 0,7 \\
\hline Primaria & 9 & 3,3 \\
\hline Secundaria & 90 & 33,1 \\
\hline Superior no universitaria & 106 & 39,0 \\
\hline Superior universitaria & 65 & 23,9 \\
\hline Total & 272 & 100,0 \\
\hline \multicolumn{3}{|l|}{ Cargo } \\
\hline Dueño & 263 & 96,7 \\
\hline Administrador & 8 & 3,0 \\
\hline Gerente & 1 & 0,3 \\
\hline Total & 272 & 100,0 \\
\hline \multicolumn{3}{|c|}{ Tiempo que desempeña en el cargo } \\
\hline 1 a 3 & 93 & 34,2 \\
\hline 4 a 6 & 135 & 49,6 \\
\hline 7 a más & 44 & 16,2 \\
\hline Total & 272 & 100,0 \\
\hline
\end{tabular}

Fuente: Cuestionario aplicado a los representantes de las mypes del distrito de Chimbote periodo 2010-2013. 
Tabla 2. Características de las mypes del distrito de Chimbote, periodo 2010- 2013.

\begin{tabular}{|c|c|c|}
\hline De la empresa: & Número de mype & Porcentaje \\
\hline \multicolumn{3}{|c|}{ Tiempo de permanencia de la empresa en el rubro } \\
\hline 0 a 3 & 81 & 29,8 \\
\hline 4 a 6 & 100 & 36,7 \\
\hline 7 a más & 91 & 33,5 \\
\hline Total & 272 & 100,0 \\
\hline \multicolumn{3}{|l|}{ La empresa es: } \\
\hline Formal & 192 & 70,6 \\
\hline Informal & 80 & 29,4 \\
\hline Total & 272 & 100,0 \\
\hline \multicolumn{3}{|c|}{ Su empresa se creó para: } \\
\hline Generar ganancia & 209 & 76,8 \\
\hline Subsistencia & 63 & 23,2 \\
\hline Total & 272 & 100,0 \\
\hline
\end{tabular}

Fuente: Cuestionario aplicado a los representantes de las mypes del distrito de Chimbote, periodo 2010-2013

Tabla 3. Formalización de las mypes del distrito de Chimbote, periodo 2010- 2013.

\begin{tabular}{lcc}
\hline De la formalización: & Número de mype & Porcentaje \\
\hline ¿La formalización le proporciona ventaja a su empresa? & & \\
\hline Sí & 56 & 55,0 \\
No & 120 & 45,0 \\
Total & & 100,0 \\
\hline ¿Qué ventajas proporciona su empresa? & 43 & 35,8 \\
\hline Aumento de ventas & 69 & 57,5 \\
Obtención de créditos & 8 & 6,7 \\
Otros & 120 & 100,0 \\
Total & & \\
\hline ¿En qué régimen está constituida su empresa? & 66 & 55,0 \\
\hline General & 54 & 45,0 \\
Específico & 0 & 0,0 \\
Simplificado & 120 & 100,0 \\
Total & & \\
\hline ¿Qué tipo de comprobante emite? & 58 & 48.3 \\
\hline Boletas & 62 & 51.7 \\
Facturas & 120 & 100,0 \\
\hline Total & & \\
\hline ¿Cree usted que la formalización le ayuda en el mercado? & 119 & 99,2 \\
\hline Sí & 1 & 0,8 \\
\hline No & 120 & 200,0 \\
\hline Total & $2010-2013$
\end{tabular}

Fuente: Cuestionario aplicado a los representantes de las mypes del distrito de Chimbote, periodo 2010-2013. 
Tabla 4. Características del financiamiento en las mypes del distrito de Chimbote, periodo 2010- 2013.

\begin{tabular}{|c|c|c|}
\hline Del financiamiento & Número de mypes & Porcentaje \\
\hline \multicolumn{3}{|c|}{ ¿La empresa ha solicitado crédito financiero? } \\
\hline Sí & 196 & 100,0 \\
\hline No & 0 & 0,0 \\
\hline Total & 196 & 100,0 \\
\hline \multicolumn{3}{|c|}{ ¿Cuántas veces ha solicitado un crédito? } \\
\hline 1 a 3 veces & 56 & 28,6 \\
\hline 4 a 5 veces & 72 & 36,7 \\
\hline 6 a más veces & 68 & 34,7 \\
\hline Total & 196 & 100,0 \\
\hline \multicolumn{3}{|c|}{ ¿A qué tipo de institución financiera? } \\
\hline Bancaria & 153 & 78,0 \\
\hline No bancaria & 43 & 22,0 \\
\hline Usureros & 0 & 0,0 \\
\hline Ninguno & 0 & 0,0 \\
\hline Total & 196 & 100,0 \\
\hline \multicolumn{3}{|l|}{ ¿Qué tasa de interés pagó? } \\
\hline Menos del $20 \%$ anual & 70 & 35,7 \\
\hline Más de $20 \%$ anual & 126 & 64,3 \\
\hline No solicitó crédito & 0 & 0,0 \\
\hline Total & 196 & 100,0 \\
\hline \multicolumn{3}{|c|}{ ¿En qué ha invertido el crédito recibido? } \\
\hline Capital de trabajo & 170 & 86,7 \\
\hline Compra de activos & 17 & 8,7 \\
\hline Mejoramiento de la infraestructura & 9 & 4,6 \\
\hline No solicitó crédito & 0 & 0 \\
\hline Total & 196 & 100,0 \\
\hline
\end{tabular}

Fuente: Cuestionario aplicado a los representantes de las mypes del distrito de Chimbote periodo 2010-2013

Tabla 5. Características de la capacitación de las mypes del distrito de Chimbote, periodo 2010- 2013.

\begin{tabular}{lcc}
\hline De la capacitación & Número de mypes & Porcentaje \\
\hline Se ha capacitado en los últimos años & & \\
\hline Sí & 74 & 96,1 \\
No & 3 & 3,9 \\
Total & 77 & 100,0 \\
\hline Cuantas veces se ha capacitado & & \\
\hline 1-2 veces & 64 & 83,1 \\
3 a más veces & 13 & 16,9 \\
Total & 77 & 100,0 \\
\hline
\end{tabular}




\begin{tabular}{lcc}
\hline Para usted la capacitación es una... & & \\
\hline Inversión & 56 & 72,7 \\
Gasto & 21 & 27,3 \\
Total & 77 & 100,0 \\
\hline ¿Qué tipo de capacitación ha recibido su personal & & \\
\hline Curso en TIC & 0 & 0,0 \\
Marketing & 65 & 84,4 \\
competitividad y emprendimiento & 3 & 3,9 \\
Atención al cliente & 9 & 11,7 \\
No capacita a su personal & 0 & 0,0 \\
Total & 77 & 100,0
\end{tabular}

Fuente: Cuestionario aplicado a los representantes de las mypes del distrito de Chimbote, periodo 2010-2013

Tabla 6. Competitividad de las mypes del distrito de Chimbote, periodo 2010- 2013.

\begin{tabular}{|c|c|c|}
\hline De la competitividad & Número de mypes & Porcentaje \\
\hline \multicolumn{3}{|c|}{ ¿Cuenta con un plan estratégico? } \\
\hline Sí & 52 & 67,7 \\
\hline No & 26 & 33,3 \\
\hline Total & 78 & 100,0 \\
\hline \multicolumn{3}{|c|}{ ¿Conoce usted el término competitividad? } \\
\hline Sí & 71 & 91,0 \\
\hline No & 7 & 9,0 \\
\hline Total & 78 & 100,0 \\
\hline \multicolumn{3}{|c|}{ ¿Cree que su empresa es competitiva? } \\
\hline Sí & 74 & 94,9 \\
\hline No & 4 & 5,1 \\
\hline Total & 78 & 100,0 \\
\hline \multicolumn{3}{|c|}{ ¿Conoce usted a sus principales competidores? } \\
\hline Sí & 39 & 50,0 \\
\hline No & 8 & 10,3 \\
\hline Algunos & 31 & 39,7 \\
\hline Total & 78 & 100,0 \\
\hline \multicolumn{3}{|c|}{ ¿Ha mejorado la infraestructura de su empresa? } \\
\hline Sí & 77 & 98,7 \\
\hline No & 1 & 1,3 \\
\hline Total & 78 & 100,0 \\
\hline \multicolumn{3}{|c|}{ ¿De qué forma es competitiva su empresa? } \\
\hline Precios bajos & 36 & 22,0 \\
\hline Calidad del producto & 36 & 44,0 \\
\hline Atención al cliente & 6 & 34,0 \\
\hline Total & 78 & 100,0 \\
\hline
\end{tabular}

Fuente: Cuestionario aplicado a los representantes de las mypes del distrito de Chimbote periodo 2010- 
Tabla 7. Rentabilidad de las mypes del distrito de Chimbote, periodo 2010-2013.

\begin{tabular}{lcc}
\hline De la rentabilidad & Número de mype & Porcentaje \\
¿Conoce usted el término de rentabilidad? & 125 & \\
Sí & 3 & 97,7 \\
No & 128 & 2,3 \\
Total & & 100,0 \\
¿Percibe que su empresa es rentable? & 115 & \\
Sí & 13 & 89,8 \\
No & 128 & 10,2 \\
Total & 115 & 100,0 \\
¿Cree usted que ha mejorado la rentabilidad de su empresa? & \\
Sí & 13 & 89,8 \\
No & 128 & 10,2 \\
Total & & 100,0 \\
¿Utiliza registros contables? & 125 & \\
Si & 3 & 97,4 \\
No & 128 & 2,3 \\
Total & 100,0
\end{tabular}

¿Ha mejorado la infraestructura de su empresa con la rentabilidad que obtuvo?

$\begin{array}{lcc}\mathrm{Si} & 115 & 89,8 \\ \text { No } & 13 & 10,2 \\ \text { Total } & 118 & 100,0\end{array}$

Fuente: Cuestionario aplicado a los representantes de las mypes del distrito de Chimbote, periodo 2010-2013.

\section{DISCUSIÓN}

Con respecto a los representantes de las mypes (Tabla 1), se determinó como resultado que la edad promedio de los representantes está entre 31 a 50 años en un 51,6\%, de 18 a 30 años en un $27,0 \%$ y de 51 a más años solo alcanzó un 21,4\% dejando entrever que las mypes están siendo dirigidas por personas adultas (73\%); entre los representantes de las mypes prevalece el sexo masculino con un $63,6 \%$ sobre el femenino, que solo tiene el $36,4 \%$. A la pregunta sobre el grado de instrucción de los representantes se estableció que tienen un grado de instrucción superior no universitaria y secundaria $(72,1 \%)$; a su vez, algunos representantes tiene instrucción primaria y superior universitaria $(27,2 \%)$ y solo un pequeño porcentaje no tiene instrucción $(0,7 \%)$. Con respecto a la pregunta de qué cargo desempeña, el representante de la mype en su mayoría es el dueño (96,7 \%) y solo son administradores o gerentes el (3,3\%). Sobre el tiempo que desempeña en el cargo se obtuvo como resultado que los representantes de las mypes al ser los dueños que dirigen sus propias mype desempeña el cargo de 4 a más de 7 años (65.8 \%) de acuerdo al tiempo de funcionamiento que tiene su mype. 
Con respecto a las mypes (Tabla 2), a la pregunta de tiempo de permanencia en el rubro, el resultado indica que la mayoría de mypes tiene una permanencia promedio en el mercado de 4 años a más de 7 (70,2\%), que indica que ha sido gestionada adecuadamente; y solo una minoría tiene una permanencia de 0 a 3 años $(29,8 \%)$ el presente estudio determinó que la mayoría de las mypes son formales ( 70,6\%), que les permite funcionar de acuerdo con la normatividad, obteniendo los beneficios correspondientes; pero también se ha obtenido como resultado que existen una minoría de mype que son informales ( 29,4\%); y a la pregunta para qué se creó la empresa, el resultado indica que la mayoría de las mypes fueron creadas para generar ganancias (76,8\%), que es el fin de toda empresa y solo una minoría se creó como subsistencia $(23,2 \%)$ a falta de un empleo para la familia.

Con respecto a la formalización (Tabla ) a las preguntas si la formalización le proporciona ventajas a su empresa y qué ventajas, respondieron que sí le proporciona ventajas (55\%), en alusión a que, al estar formalizada, tiene el respaldo de la normatividad al realizar contratos públicos y privados entre otras ventajas, como obtenciones de créditos de las entidades financieras y aumento en sus ventas (93,3\%). A la pregunta sobre en qué régimen está constituida su empresa, la mayoría se encuentra en el régimen general (55\%) y en simplificado ( $45 \%$ ) por la misma característica del régimen a que pertenecen estas emiten en su mayoría facturas (51,7 \%) como comprobante de ventas. Y solo una minoría emite facturas (48,3\%). La mayoría de las mypes asegura que la formalización le ayuda a posicionarse en el mercado $(99,2 \%)$ y solo para algunas mype la formalización no le ayuda a posesionarse en el mercado $(0,8 \%)$, por lo que algunos permanecen en la informalidad. Para Loayza (2008) el mecanismo de la formalización es sumamente importante por las consecuencias que tiene en el empleo, la eficiencia y el crecimiento económico. La informalidad surge cuando los costos de circunscribirse al marco legal y normativo de un país son superiores a los beneficios que ello conlleva. La formalidad involucra costos tanto al ingresar a este sector en complejos y costosos procesos de inscripción y registro -como de permanecer dentro-, con pago de impuestos, cumplir las normas referidas a beneficios laborales y remuneraciones, manejo ambiental, salud, pero con la posibilidad de expandirse a mercados tanto locales como internacionales.

Con respecto al financiamiento (Tabla 4), la totalidad de mypes del distrito de Chimbote, para mantener sus operaciones en el mercado, ha solicitado créditos financieros (100\%) y de 1 a 5 veces $(65,3 \%)$ y ha solicitado crédito más de 6 veces $(34,7 \%)$, ha solicitado crédito al sistema bancario y al sistema no bancario (100\%) que les han cobrado intereses altos, es decir, más del 20\% anual (64,3\%). Estos créditos han sido utilizados como capital de trabajo $(86,7 \%)$ y solo una minoría lo ha utilizado en compra de activos y mejoramiento de su infraestructura (13,3\%). El financiamiento considerado como el conjunto de recursos monetarios financieros para realizar una actividad económica, generalmente se trata de sumas tomadas a préstamo que complementan los recursos propios. El financiamiento se contrata dentro o fuera del país a través de créditos, empréstitos y otras obligaciones derivadas de la suscripción o emisión de títulos de crédito o cualquier otro documento pagadero a plazo. Las mypes del rubro en estudio consideran que la falta de acceso al crédito es uno de los obstáculos más importantes para desarrollar sus negocios 
seguido de la inflación, impuestos, trámites, regulaciones, corrupción e inseguridad. Las altas tasas de interés, las exigencias para constituir garantías, y la falta de acceso a los mercados de capital y el financiamiento de largo plazo constituyen los principales problemas identificados por parte de las mypes. Este problema afecta especialmente a las pequeñas empresas que son demasiado grandes para las microfinanzas y demasiado pequeñas para los préstamos bancarios, lo cual permite insuficiencia para invertir o inversiones innecesarias.

Con respecto a la capacitación (Tabla 5). Las mypes del distrito de Chimbote en los últimos años ha capacitado a su personal (96,1\%), esto ha permitido su crecimiento y desarrollo; se han capacitado de 1 a 2 veces $(83,1 \%)$, porque consideran que la capacitación es una inversión, lo cual así lo manifiesta una gran mayoría $(72,7$ \%) y solo una minoría $(27,3 \%)$ lo considera un gasto. Es por eso que todavía existen algunas mypes que no pueden mantenerse competitivas en el mercado. Las mypes del distrito de Chimbote capacitan a su personal en marketing y atención al cliente (96,1 \%) y en competitividad y emprendimiento solo el (3,9\%). Según Vizacarra (2009), la capacitación se refiere a los métodos que se usan para proporcionar a las personas, dentro de la empresa, las habilidades que necesitan para realizar su trabajo. Este es un proceso que lleva a la mejora continua y con esto a implantar nuevas formas de trabajo; las mypes que lo adopte le generará un valor agregado volviéndolas competitivas.

Con respecto a la competitividad (Tabla 6), las mypes del distrito de Chimbote para permanecer en el mercado deben ser competitivas. Es por eso que el presente estudio trata de determinar las características de la competitividad y cómo se presenta esta variable en las mypes, teniendo como resultado que en la mayoría de las mypes se conoce el término competitividad (91\%), además creen que son competitivas (94,9\%), por la calidad de sus productos $(46,2 \%)$, por sus precios bajos $(46,2 \%)$ y por la buena atención al cliente $(7,6 \%)$, esto debido a que conocen las debilidades y fortalezas de sus principales competidores (89\%); y, además, han mejorado su infraestructura (98,7\%) que les da una buena presentación e imagen hacia los clientes, que les permite ser competitivos a pesar de que un poco más de la mitad no cuentan con un plan estratégico $(67,7 \%)$. Para Vásquez (2011) competitividad es la capacidad de una organización pública o privada, lucrativa o no, de mantener sistemáticamente ventajas comparativas, es decir, sus habilidades, recursos, conocimientos y atributos que le permitan alcanzar, sostener y mejorar una determinada posición en el entorno socioeconómico, pero también es importante tener en cuenta que en el contexto de competitividad tanto nacional como internacional, las mypes en estudio no solo tienen la necesidad de ser competitivas internamente, sino que la globalización exige buscar la competitividad externa y consolidarse haciendo alianzas estratégicas lo cual están logrando.

Con respecto a la rentabilidad (Tabla 7), la mayoría de las mypes del distrito de Chimbote que permanecen en el mercado de 4 a más de 7 años, que son competitivas, que usan adecuadamente los créditos financieros, que consideran a la capacitación como una inversión para lograr su competitividad, conoce los términos de rentabilidad (97,7\%), percibe que su empresa es rentable y mejora constantemente esa rentabilidad $(89,8 \%)$ y lo corrobora, porque una gran mayoría utiliza registros contables y ha mejorado su infraestructura 
$(89,8 \%)$ que le permite presencia y participación competitiva en el mercado. Según Acosta (2005), la rentabilidad es la relación que existe entre la utilidad y la inversión necesaria para lograrla, la cual mide la efectividad de la gerencia de una empresa, demostrada por las utilidades obtenidas de las ventas realizadas y la utilización de las inversiones, y su competitividad para lograr utilidades. Dichas utilidades, a su vez, son la conclusión de una administración competente, una planeación inteligente, y una reducción integral de costos y gastos, los cuales si se está realizando en las mypes en estudio.

\section{CONCLUSIONES}

El presente estudio permite concluir que:

- La mayoría de los representantes de las mypes del distrito de Chimbote, periodo 2010-2013, son adultos y tienen una edad promedio entre 31 a 50 años, son de sexo masculino, con grado de instrucción superior no universitaria, se desempeñan en las mypes como dueños y como tal está en el cargo de 4 años a más de 7 años.

- La mayoría de las mypes del distrito de Chimbote tienen en el rubro una permanencia de 4 años a más de 7 años, son formales y fueron creadas para obtener ganancias.

\section{Con respecto a la formalización}

- La mayoría de las mypes del distrito de Chimbote, asegura que la formalización les proporciona ventajas como obtención de créditos, aumento de sus ventas y ayudándoles a posesionarse en el mercado.

\section{Con respecto al financiamiento}

- La totalidad de las mypes del distrito de Chimbote utiliza el financiamiento y ha solicitado créditos de 1 a 5 veces, pagando una tasa de más del 20 \% anual, invirtiéndolo en capital de trabajo.

\section{Con respecto a la capacitación}

- La mayoría de las mypes consideran que la capacitación es una inversión, y ha capacitado a su personal más de una vez en marketing y atención al cliente.

\section{Con respecto a la competitividad}

- La mayoría de las mypes cuenta con un plan estratégico, conoce a sus competidores y percibe que es competitiva.

\section{Con respecto a la rentabilidad}

- La mayoría de las mypes del distrito de Chimbote percibe que es rentable, por lo mismo que con esa rentabilidad ha mejorado su infraestructura. 


\section{REFERENCIAS BIBLIOGRÁFICAS}

Acosta, J. (2002). Análisis e interpretación de la información financiera. Consultado el 12 de febrero del 2013. Disponible en: http://www.gestiopolis.com/recursos/experto/catsexp/pagans/fin/37/rentabiyproduct.htm

Arruñada, B. (2007). El imperio de eficiencia en la formalización de empresas, 1, 106-107.

Ayala, S. (2004). Proceso de desarrollo de los recursos humanos. Consultado el 15 de diciembre del 2012. Disponible en: http://www.elprisma.com/apuntes/ administracion_de_empresas/capacitacionrecursoshumanos/

Loayza, N. (2008). Causas y consecuencias de la informalidad en el Perú. Consultado el 20 de diciembre 2013. Disponible de: www.bcrp.gob.pe/docs/Publicaciones/RevistaEstudios-Economicos/15/Estudios-Economicos-15-3.pdf

Ponjuán, G. (2010). Introducción de las técnicas gerenciales en la práctica de las organizaciones de información. Consultado el 7 de diciembre del 2013. Disponible en: http://www. congreso-info.cu/Userfiles/File/Info/Info97/ponencias/187.pdf

Tantas, L. (2010). Caracterización del financiamiento, la capacitación y la rentabilidad de las mypes del sector comercio. Rubro distribuidoras de útiles de oficina del distrito de Chimbote, periodo 2008-2009. Proyecto de tesis para optar el grado de Licenciado en Administración, Escuela de Administración, Universidad Católica Los Ángeles de Chimbote, Chimbote, Perú.

Torres, O. (2010). Financiamiento a mypes. [En línea]. Consultado el 16 de enero del 2013. Disponible en: http://otorres.blogdiario.com/

Vásquez, I. (2011). Formalización empresarial. Mi empresa en marcha. Consultada el 1 noviembre de 2011. Disponible en: http://miempresaenmarcha.blogspot. com/2011/08/formalizacion-empresarial.html

Vela, L. (2007). Caracterización de las micro y pequeñas empresas (mypes) en el Perú y desempeño de las micro finanzas. Universidad Nacional Pedro Ruiz Gallo, Lambayeque, Perú.

Vélez, A. (2010). Guía de constitución y formalización de empresas. Consultado el 25 de agosto del 2013. Disponible en: https://alexvelez.wordpress.com/category/ebooks/ page/2/

Vigil, Y. (2008). Formación, crecimiento y proyección de las MyPES (Perú). [En línea]. Consultado el 16 de enero del 2013. Disponible en: http://www.monografias.com/ trabajos58/mypes/mypes.shtml.

Vizacarra, N. (2009). Importancia de la capacitación para las MyPES. Mundo MyPE. Extraído el 3 febrero, 2013, de http://www.mundomype.com/articulos/44-recursoshumanos/167-importancia-de-la-capacitacion-para-las-mype.htmllo 